Acta Crystallographica Section E

Structure Reports

Online

ISSN 1600-5368

\section{B. H. M. Mruthyunjayaswamy,} Omkar B. Ijare, ${ }^{a} \mathrm{Y}$ Jadegoud, ${ }^{a}$

A. Ray Chaudhurib and T. N. Guru Row ${ }^{b_{*}}$

a Department of Chemistry, University of Gulbarga, Gulbarga, Karnataka 585 106, India, and ${ }^{\mathbf{b}}$ Solid State and Structural Chemistry Unit, Indian Institute of Science, Bangalore, Karnataka 560012 , India

Correspondence e-mail: ssctng@sscu.iisc.ernet.in

\section{Key indicators}

Single-crystal X-ray study

$T=293 \mathrm{~K}$

Mean $\sigma(\mathrm{C}-\mathrm{C})=0.004 \AA$

$R$ factor $=0.037$

$w R$ factor $=0.084$

Data-to-parameter ratio $=16.8$

For details of how these key indicators were automatically derived from the article, see http://journals.iucr.org/e.

\title{
Ethyl N-(5-bromo-3-phenylindol-2-yl)carbamate
}

The crystal structure of the title compound, $\mathrm{C}_{17} \mathrm{H}_{15} \mathrm{BrN}_{2} \mathrm{O}_{2}$, has been determined at room temperature. The indole moiety is essentially planar and the structure is stabilized by intra- and intermolecular $\mathrm{N}-\mathrm{H}$. . O hydrogen bonds.

\section{Comment}

The title compound, (I), shows $80 \%$ antifilerial activity in vitro against $O$. Gutturosa (Mruthyunjayaswamy et al., 2002). The indole moiety is planar. The activity is due to the presence of the ethyl carbamate moiety. Hydrogen bonds are given in Table 1.

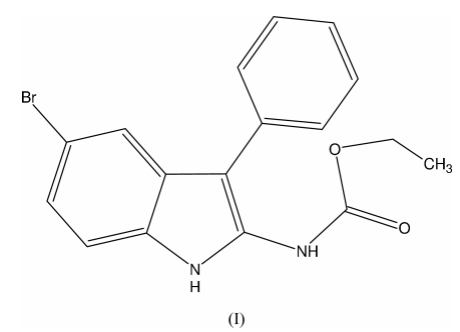

\section{Experimental}

The title compound was prepared by nitrosation of 5-bromo-3phenylindole-2-carboxyhydrazide followed by the resulting product in ethanol for $5 \mathrm{~h}$ (Hiremath et al., 1978). Crystals were grown from ?tpbgc $=^{\wedge}$ st_head3_bgcolour] $>$ 2-propanol.

Crystal data

$\mathrm{C}_{17} \mathrm{H}_{15} \mathrm{BrN}_{2} \mathrm{O}_{2}$

$M_{r}=359.21$

Monoclinic, $P 2_{1} / n$

$a=13.602(2) \AA$

$b=10.1781(17) \AA$

$c=23.565$ (4) $\mathrm{A}$

$\beta=106.267(3)^{\circ}$

$V=3131.8(9) \AA^{3}$

$Z=8$

$$
\begin{aligned}
& D_{x}=1.524 \mathrm{Mg} \mathrm{m}^{-3} \\
& \text { Mo } K \alpha \text { radiation } \\
& \text { Cell parameters from } 9585 \\
& \quad \text { reflections } \\
& \theta=2.2-23.3^{\circ} \\
& \mu=2.63 \mathrm{~mm}^{-1} \\
& T=293(2) \mathrm{K} \\
& \text { Prism, pale brown } \\
& 0.32 \times 0.21 \times 0.10 \mathrm{~mm}
\end{aligned}
$$

\section{Data collection}

Bruker SMART CCD area-detector diffractometer

$\varphi$ and $\omega$ scans

Absorption correction: multi-scan

(XPREP; Bruker, 1998)

$T_{\min }=0.514, T_{\max }=0.769$

39464 measured reflections

Refinement

Refinement on $F^{2}$

$R\left[F^{2}>2 \sigma\left(F^{2}\right)\right]=0.037$

$w R\left(F^{2}\right)=0.084$

$S=0.87$

6698 reflections

399 parameters
6698 independent reflections 4047 reflections with $I>2 \sigma(I)$

$R_{\text {int }}=0.061$

$\theta_{\max }=27.4^{\circ}$

$h=-17 \rightarrow 17$

$k=-13 \rightarrow 13$

$l=-30 \rightarrow 28$

$\mathrm{H}$-atom parameters constrained $w=1 /\left[\sigma^{2}\left(F_{o}^{2}\right)+(0.0456 P)^{2}\right]$

where $P=\left(F_{o}^{2}+2 F_{c}^{2}\right) / 3$

$(\Delta / \sigma)_{\max }=0.001$

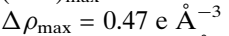

$\Delta \rho_{\min }=-0.38 \mathrm{e}^{-3}$
Received 15 May 2002 Accepted 6 June 2002 Online 14 June 2002 


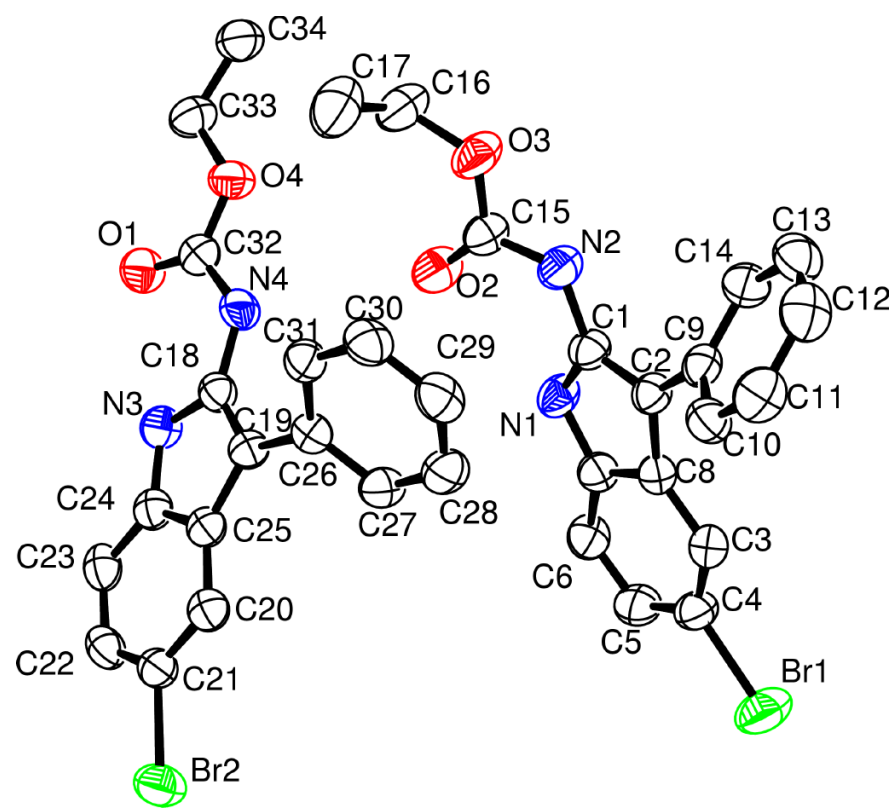

Figure 1

The asymmetric unit of (I), with the atom-numbering scheme. Displacement ellipsoids are drawn at the $50 \%$ probability level. $\mathrm{H}$ atoms have been omitted for clarity.

\section{Table 1}

Hydrogen-bonding geometry $\left(\AA{ }^{\circ}\right)$.

\begin{tabular}{lllll}
\hline$D-\mathrm{H} \cdots A$ & $D-\mathrm{H}$ & $\mathrm{H} \cdots A$ & $D \cdots A$ & $D-\mathrm{H} \cdots A$ \\
\hline $\mathrm{N} 1-\mathrm{H} 1 \cdots \mathrm{O} 1$ & 0.86 & 2.19 & $2.722(3)$ & 120 \\
$\mathrm{~N} 1-\mathrm{H} 1 \cdots \mathrm{O} 3^{\mathrm{i}}$ & 0.86 & 2.28 & $2.926(3)$ & 132 \\
$\mathrm{~N} 2-\mathrm{H} 2 \cdots 3^{\mathrm{ii}}$ & 0.86 & 2.48 & $3.282(3)$ & 155 \\
$\mathrm{~N} 3-\mathrm{H} 3 \cdots \mathrm{O} 3$ & 0.86 & 2.33 & $2.797(2)$ & 114 \\
$\mathrm{~N} 3-\mathrm{H} 3 \cdots \mathrm{O} 1^{\mathrm{iii}}$ & 0.86 & 2.33 & $3.049(3)$ & 142 \\
$\mathrm{C} 33-\mathrm{H} 33 B \cdots \mathrm{O} 3$ & 0.98 & 2.37 & $2.739(3)$ & 102 \\
\hline
\end{tabular}

Symmetry codes: (i) $\frac{1}{2}+x, \frac{3}{2}-y, z-\frac{1}{2}$; (ii) $\frac{1}{2}-x, y-\frac{1}{2}, \frac{1}{2}-z$; (iii) $x-\frac{1}{2}, \frac{3}{2}-y, \frac{1}{2}+z$.

Data collection: SMART (Bruker, 1998); cell refinement: SMART; data reduction: SAINT (Bruker, 1998); program(s) used to solve structure: SHELXS97 (Sheldrick, 1997); program(s) used to refine

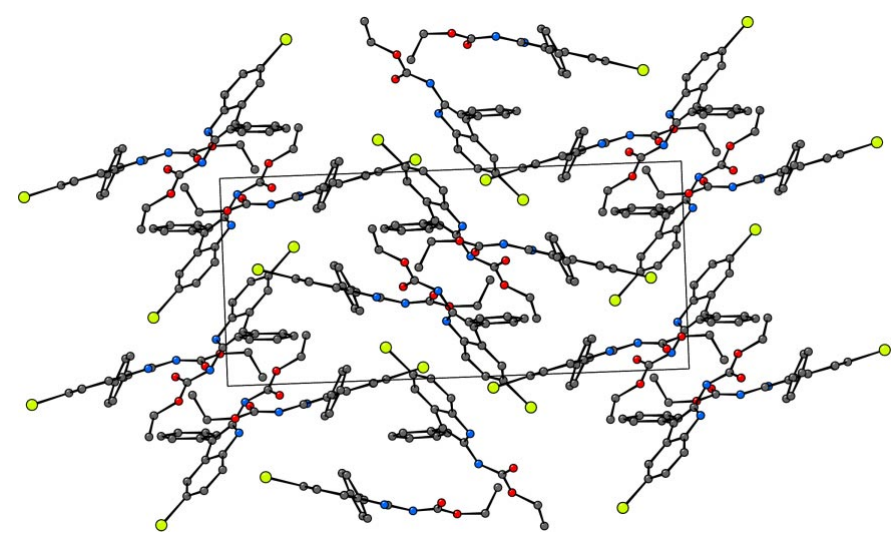

Figure 2

Packing diagram of the title compound, viewed down the $a$ axis, with $b$ vertical and $c$ horizontal. $\mathrm{H}$ atoms have been omitted for clarity.

structure: SHELXL97 (Sheldrick, 1997); molecular graphics: ORTEP-3 for Windows (Farrugia, 1997) and CAMERON (Watkin et al., 1993); software used to prepare material for publication: PLATON (Spek, 1990).

The authors thank the Department of Science and Technology, India, for data collection on the CCD facility of the IRFA-DST program, and the Chairman, Department of Chemistry, Gulbarga University, for providing facilities to carry out the synthesis of the compound.

\section{References}

Bruker (1998). SMART and SAINT. Bruker AXS Inc., Madison, Wisconsin, USA.

Farrugia, L. J. (1997). J. Appl. Cryst. 30, 565.

Hiremath, S. P., Mruthyunjayaswamy, B. H. M. \& Purohit, M. G. (1978). Indian J. Chem. Sect. B, 16, 789.

Mruthyunjayaswamy, B. H. M., Hiremath, S. P. \& Hooper, M. (2002). Unpublished results.

Sheldrick, G. M. (1997). SHELXL97 and SHELXS97. University of Göttingen, Germany.

Spek, A. L. (1990). Acta Cryst. A46, C-34.

Watkin, D. J., Pearce, L. \& Prout, C. K. (1993). CAMERON. Chemical Crystallography Laboratory, University of Oxford, England. 